\title{
Adaptive Compressed Wideband Spectrum Detection Based on Optimized Measurement Matrix
}

\author{
Yongle $\mathrm{Yu}^{1, \mathrm{a}}$, Zhengbao Zhang ${ }^{1, \mathrm{~b}}$, Chao Guo ${ }^{2, \mathrm{c}}$ and Gaofeng Fan 1,d \\ ${ }^{1}$ Department of Information Engineering, Ordnance Engineering College, Shijiazhuang 050003, China; \\ 2Unit 71777 of PLA, Jinan 250100, China. \\ ayu_yong_le@163.com, bzhengbaozhang@163.com, 'oguochaojunxie@163.com, dfanr84@sina.cn
}

Keywords: cognitive radio, compressed sensing, spectrum detection, measurement matrix.

\begin{abstract}
The emergence of compressed sensing technology brought a revolutionary opportunity to wideband spectrum sensing, which could be used to reduce the sampling rate and reconstruct the sparse spectrum. In combination of measurement matrix optimization and adaptive process of compressive sampling, a modified adaptive compressed wideband spectrum sensing algorithm is proposed. In this paper, the column vector autocorrelation of the observation matrix was reduced, and the impact of optimized matrix on reconstruction algorithm was analyzed. The simulation results show that the proposed algorithm has a lower mean square error (MSE) than that of the traditional algorithm, and the detection probability is higher at the same number of observations.
\end{abstract}

\section{Introduction}

Cognitive radio technology $(\mathrm{CR})$ is an effective means to solve the problem of spectrum shortage [1], spectrum sensing is one of the key technologies of cognitive radio. The traditional spectrum sensing method divided the wideband spectrum into several sub-bands. By using the tunable filter, spectrum sensing can detect the sub-band in time division. However, it is inflexible and unable to detect in real time. There is also a high speed analog to digital converter (ADC) for wideband signal detection, but when it is faced to the bandwidth ranged up to thousands of $\mathrm{MHz}$, it would bring the problems of large data acquisition and difficult hardware implementation.

Compressed sensing technology (CS) provides a new way to solving the problem of real-time wideband signal detection. Compressed sensing technology was first proposed by Candes ${ }^{[2]}$, Romberg, Tao ${ }^{[3]}$ and Donoho ${ }^{[4]}$ et al., which can be used to compress and sample sparse signals synchronously, and to reconstruct the signal by non-adaptive linear projection. The method of Sequential Compressed Sensing (SCS) is proposed by GU ${ }^{[5]}$. Under the premise of ensuring high probability reconstruction of the original signal, this method discusses the minimum number of observations. The basic idea of SCS is to increase the number of observations sequentially and reconstruct the signal in time to obtain a set of reconstructed signals. When the reconstructed signals of two or more adjacent groups are identical, the observation is considered as the suitable value. By improving the traditional OMP algorithm, Sparsity Adaptive Matching Pursuit (SAMP) algorithm is proposed by DO ${ }^{[6]}$, which can track and update the number of selected atoms dynamically in the process of iteration. Moreover, the algorithm introduces an atomic backtracking mechanism to ensure the reliability of the signal reconstruction results. The variable step size adaptive matching pursuit algorithm, sparsity adaptive subspace pursuit algorithm and regularized adaptive matching pursuit algorithm are proposed in [7], [8] and [9].

Adaptive algorithm is proposed to find the best reconfiguration algorithm when the sparse degree is unknown. However, all of the above studies are limited to adjusting the dimension of the projection matrix in time by the tracking method. They did not take the minimization of the column vector correlation of the projection matrices with the increased dimension into consideration. Combining the observation matrix optimization and adaptive process, the spectrum sensing algorithm of optimized adaptive compression based on observation matrix is proposed in this paper. 


\section{Compressed Sensing}

Assuming that the total bandwidth to be detected by the cognitive user is $\mathrm{B} \mathrm{Hz}$, it can be divided into L non-overlapping sub-bands evenly, and the edge position frequency of each sub-band is $f_{0}, \cdots, f_{L+1}$. As the prime user can occupy the sub-band arbitrarily, the PSD is approximately to zero when the sub-band is not occupied, and the PSD in the wideband is sparse.

Supposing that the signal to be measured is $x(t)$, the discrete value of $x(t)$ is $x(n)$, the autocorrelation function of $x(n)$ is $r(n)=E\left[x(m) x^{*}(m-n)\right], r(f)$ corresponding to the Fourier transform value of $r(n)$, which represents the power spectral density, the corresponding relationship is:

$$
r(n)=F^{-1} r(f)
$$

In (1), $F^{-1}$ represents an inverse Fourier transform. In the process of CS, $r_{y}(n)$ is obtained by the projection of autocorrelation function on a random Gaussian matrix $\Phi$, and the process can be expressed as:

$$
r_{y}=\Phi r_{x}=\Phi G Z=\Psi Z
$$

In (2), $\Psi=\Phi G$ is defined as a compressed sensing matrix. As shown in (2), as long as the sparse vector $Z$ is reconstructed from the measured value $r_{y}$, the spectrum information of the signal could be obtained. Among them, the sparse vector $Z$ can be obtained by solving the problem of $l_{1}$ norm optimization in (3)

$$
\hat{Z}=\arg \min \|z\|_{l_{1}}
$$

The greedy algorithm and convex optimization algorithm are used to solve this problem. Among them, the match pursuit algorithm convergence faster, and the experiment in this paper would use this algorithm.

\section{Observation Matrix Optimization}

RIP conditions fully guarantee the signal recovery performance of the sensing system, which plays an extremely important role in the CS field. For any $\mathrm{K}$-sparse signal $X$, if there is a constant $\delta_{K} \in(0,1)$ which could support the equation (4):

$$
\left(1-\delta_{K}\right)\|X\|_{2}^{2} \leq\|\Psi X\|_{2}^{2} \leq\left(1+\delta_{K}\right)\|X\|_{2}^{2}
$$

That the matrix satisfies the K-order RIP condition. RIP makes any column of $\Psi$ almost orthogonal, and ensures the non-correlation between $\Phi$ with G. However, the verification of RIP conditions is very difficult. In [10], Michel gave a more concise expression:

$$
\|X\|_{0}<\frac{1}{2}\left(1+\frac{1}{\mu(\Psi)}\right)
$$

In (5), $\mu(\Psi)$ denotes the degree of correlation of $\Psi$, which is defined as the normalized inner product with the largest absolute value between all columns of the perceptual matrix $\Psi$, that:

$$
\mu(\Psi)=\max _{\substack{1 \leq i, j \leq N \\ i \neq j}}\left(\frac{\left|\varphi_{i}^{H} \varphi_{j}\right|}{\left\|\varphi_{i}\right\|_{2} \cdot\left\|\varphi_{j}\right\|_{2}}\right)
$$

It can be seen from that the correlation coefficient has a significant effect on the recovery accuracy of the signal, $\mu(\Psi)$ could be sufficiently small if $\Psi$ is chosen properly, then RIP will ensure that the signal is recovered with high probability. Therefore, it is important to reduce the correlation between the columns of $\Psi$ to enhance the performance of the reconstruction algorithm.

Define a complex Gram matrix $G=\Psi^{H} \Psi$, which represents the correlation degree of matrix $\Psi$. 


$$
G=\left[\begin{array}{cccc}
g_{11} & g_{12} & \cdots & g_{1 N} \\
g_{21} & g_{22} & \cdots & g_{2 N} \\
\vdots & \vdots & \ddots & \vdots \\
g_{N 1} & g_{N 2} & \cdots & g_{N N}
\end{array}\right]
$$

In $\mathrm{G}, g_{i j}=\varphi_{i}^{H} \varphi_{j}$ represents the cross-correlation coefficient between any two columns in the $\Psi$. The most ideal case of the matrix $G$ is to become a diagonal matrix. Therefore, the ultimate goal of matrix optimization is to set the appropriate sensing matrix $\Psi$ such that the Gramm matrix $G$ has the closest form to the diagonal matrix.

\section{Adaptive Compressed Wideband Spectrum Sensing}

Adaptive compressed sensing refers to adaptively adjust the size of the observation matrix when the signal sparsity is unknown, and the key technique is to determine the termination threshold. In order to select the optimal number of observations, a sequential compressed algorithm is used to estimate the reconstruction error of the sparse vector $\hat{\theta}$. Firstly, it is assumed that the initial number of observations is $M_{0}$ according to the empirical values of the different signals. This value represents the number of row vectors of the initial projection matrix. Suppose that the number of observations increased by the step of $\mathrm{T}$, and by receiving the additional T observations, the reconstructed vector $\hat{\theta}^{M_{0}}$ is obtained. Adaptive compressed sensing dynamically adjusts the number of observations. Since there is a certain degree of redundancy between the column vectors of the projection matrix, the projection matrix cannot be determined by the adaptive process alone. Only the adaptive process of compressed sensing is combined with the optimization process of projection matrix, would the optimal projection matrix be determined to achieve the best spectrum detection effect.

In summary, this paper proposes a sparsity adaptive matching pursuit-optimized algorithm for wideband detection. The algorithm is described as follows:

Input: Wideband spectrum signal

Output: Sub-band occupation information

Step 1: Generate $\varphi_{0}$, which is a $M_{0} \times N$ dimensional random matrix. Combining the Fourier basis $\mathrm{F}$ with the wavelet basis $\mathrm{W}$, the compressed sensing matrix $\psi_{0}=\varphi_{0} F^{-1} W^{-1}$ is obtained;

Step 2: Calculate the Gram matrix of $\psi_{0}: G=\tilde{\psi}_{0}^{T} \tilde{\psi}_{0} \cdot \tilde{\psi}_{0}$ Is a matrix which column vectors are normalized of $\psi_{0}$;

Step 3: Set the iteration number Q and threshold $\gamma \cdot \hat{g}_{i, j}= \begin{cases}\gamma g_{i, j} & \left|g_{i, j}\right| \geq t \\ \gamma t . \operatorname{sign}\left(g_{i, j}\right) & t>\left|g_{i, j}\right| \geq \gamma t \text { Is used for } \\ g_{i, j} & \gamma t>\left|g_{i, j}\right|\end{cases}$ the singular value decomposition (SVD) of the elements $g_{i, j}$ of matrix G. To obtain $\hat{G}$, the elements from $\mathrm{k}+1$ to $\mathrm{N}$ of the diagonal matrix are set to zero and repeated until the iterative process is completed;

Step 4: Let matrix $\hat{G}$ subject to SVD decomposition, and construct matrix $D=\sqrt{S_{0}} U_{0}^{T}$, which is the optimized compressed sensing matrix. Among them, $S_{0}$ is the first k SVD values of $\mathrm{S}$, and $U_{0}$ is the first $\mathrm{k}$ columns of $\mathrm{U}$;

Step 5: If $C_{T} d\left(\hat{Z}^{M_{0}}, H_{M_{0}+T}\right)<\lambda$ jump to step 7; if not, jump to step 6;

Step 6: increase the observation to obtain the new observation matrix $\Phi_{1}$ and the compressed sensing matrix $\psi_{1}$, and $\psi_{1}$ is optimized according to the step 3; 
Step 7: The sparse vector $\mathrm{Z}$ is reconstructed, and the power spectrum is obtained by transforming to the frequency domain, and the occupancy of the sub-band is analyzed according to the value of the power spectrum.

\section{Simulations}

In this paper, MATLAB platform is used to simulate and verify the wideband spectrum detection. During the detection process, $x(t)$ is used as the source signal, which is superimposed by a plurality of center frequency signals.

$$
x(t)=\sum_{i=1}^{5} \sqrt{E_{i} B} \sin c\left(B\left(t-\tau_{i}\right)\right) \cos \left(f_{i}\left(t-\tau_{i}\right)\right)
$$

The bandwidth of each sub-band is $\mathrm{B}=8 \mathrm{MHz}, E_{i}$ refers to the random amplitude value, $\tau_{i}$ refers to a random time offset, and $f_{i}$ refers to the center frequency of the band randomly occupied. A total of 16 sub-bands, of which 5 sub-bands are randomly occupied, the signal amplitude of these bands are subjected to normal distribution, the remaining sub-bands are blank. Supposed that the dimension of the signal to be measured is $\mathrm{N}=128$, which is the number of the Nyquist sampling during the observation period. The error threshold of reconstruction algorithm is $\zeta=10^{-2}$, and repeat the operation 1000 times to obtain the average value.

In order to represent the error between original data and reconstructed spectrum data, Mean-square Errors (MSE) is defined:

$$
M S E=\frac{E\left(\sum\left|\hat{r}_{i, j}-r_{i, j}\right|^{2}\right)}{E\left(\sum\left|r_{i, j}\right|^{2}\right)}
$$

The OMP algorithm and the SAMP algorithm are used in this experiment. According to the power spectrum obtained in step 7 of the SAMP-OP algorithm, the energy of each sub-band can be obtained. By comparing the energy value with the preset threshold value, the spectrum occupancy can be judged.

Set $M=64$, and SNR ranged from 0 20dB. The MSE of each algorithm at different SNR is shown in Fig. 1. The range of the number of observation is 30 120, and the SNR is $10 \mathrm{~d} B$, and the average value is obtained by repeating 1000 times for each observation, as shown in Fig. 2. As can be seen from Fig. 1 each curve decreases with the increase of SNR. The reconstruction performance of SAMP is better than that of OMP, and the performance is improved after the optimization of projection matrix. In Fig. 1, the MSE decreases as the number of observations increase. When there are more observations, SAMP has obvious advantages over the OMP algorithm. In general, the MSE of the optimized projection matrix in Fig. 1 and Fig. 2 are lower than that in the case of no optimization, which show that the optimized projection matrix is with higher precision.

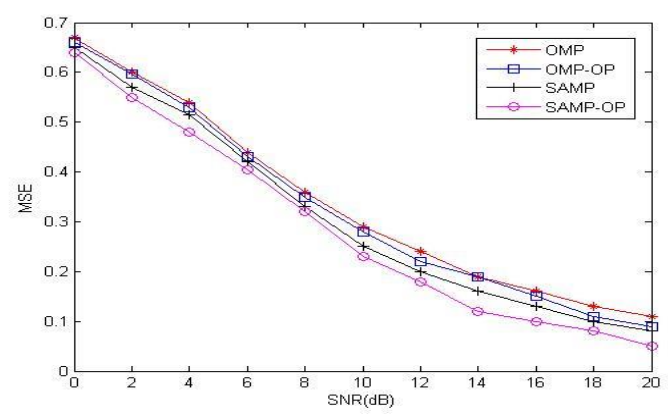

Fig. 1 MSE of different SNR 


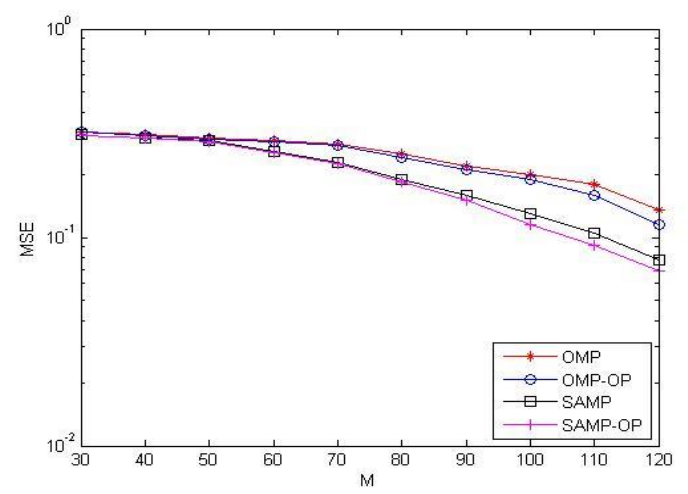

Fig. 2 MSE of different observation times

In order to verify the improvement of spectrum sensing, the relationship between detection probability and observation times was analyzed, as shown in Fig. 3. Set SNR=10dB, and spectrum holes are determined by energy detection. The occupation of the spectrum holes can be determined by the comparison of the energy value with the preset threshold.

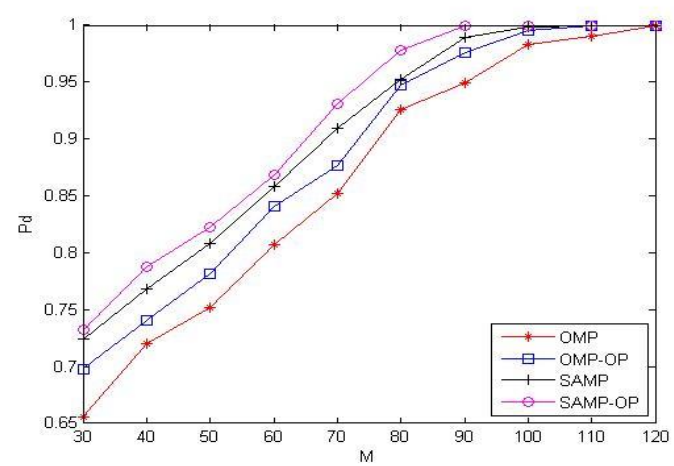

Fig. $3 \mathrm{P}_{\mathrm{d}}$ of different observation times

It can be seen from the Fig. 3 that $P_{d}$ is increased in pace with the raise of observation. For the same reconstruction algorithm, the detection probability of the projection matrix is higher than that of the matrix without optimization.

\section{Conclusion}

In the cognitive radio system, the SU cannot predict the spectrum occupancy of the PU, which the spectrum sparsity is unpredictable. The adaptive compressed sensing technology can solve this problem effectively. In this paper, a sparsity adaptive matching pursuit optimized algorithm in wideband detection is proposed, which reduce the autocorrelation between the projection matrix vectors. The adaptive adjustment of the observation is realized by estimating the reconstruction error of the sparse vector. At the end of this paper, the SAMP-OP algorithm is compared with the traditional algorithms. The simulation results show that the proposed algorithm has a lower MSE than that of the traditional algorithm, and the detection probability is higher at the same number of observations. In this paper, the technique is applied to the wideband spectrum detection in cognitive radio, and the corresponding solution and reconstruction algorithm are put forward.

\section{References}

[1]. MITOLA J. Cognitive Radio: Making Software Radios More Personal [J]. IEEE Personal Communications. Vol. 6 (1999) No. 4, p. 13-18.

[2]. CANDES E, ROMBERG J, TAO T. Stable Signal Recovery from Incomplete and Inaccurate Measurements [J]. Communications on Pure and Applied Mathematics. Vol. 59 (2006) No. 8, p. 1207-1223. 
[3]. CANDES E, ROMBERG J. Quantitative Robust Uncertainty Principles and Optimally Sparse Decompositions [J]. Foundations of Compute Math. Vol. 6 (2006) No. 4, p. 13-18.

[4]. DONOHO D L. Compressed Sensing [J]. IEEE Transactions on Information Theory. Vol. 52 (2006) No. 4, p. 1289-1306.

[5]. GU B, YANG Z, HU H F. Adaptive Wideband Spectrum Detection Based on Sequential Compressed Sensing [J]. Chinese Journal of Scientific Instrument. Vol. 32 (2011) No. 6 p. 1272-1277.

[6]. Do T T, Gan L, Nguyen N, et al. Sparsity Adaptive Matching Pursuit Algorithm for Practical Compressed Sensing[C]. The 42" Conference on Signals, Systems and Computers. Asilomar, 2008, p. 581-587.

[7]. Gao R, Zhao R Z, Hu S H. Variable Step Size Adaptive Matching Pursuit Algorithm for Image Reconstruction Based on Compressive Sensing[J]. Acta Optica Sinica. Vol. 30 (2010) No. 6, p. 1639-1644.

[8]. Yang C, Feng W, Feng H, et al. A Sparsity Adaptive Subspace Pursuit Algorithm for Compressive Sampling [J], Acta Electronica Sinica. Vol. 38(2010) No. 8, p. 1914-1917.

[9]. Liu Y X, Zhao R Z, Hu S H, et al. Regularized Adaptive Matching Pursuit Algorithm for Signal Reconstruction Based on Compressive Sensing [J]. Journal of Electronics \& Information Technology. Vol. 32 (2010) No. 11, p. 2713-2717.

[10]. Michael I E. Optimized Projections for Compressed Sensing [J]. IEEE Transactions on Signal Processing. Vol. 55 (2007) No. 12, p. 5695-5702.

[11]. Wang Z W, Xu Y T, Jiang H, et al. Design and Implementation of Wideband Spectrum Sensing System Based on USRP Platform [J]. Communications Technology. Vol. 48 (2015) No. 6, p. 750-754. 